\title{
Organocatalytic Discrimination of Non-Directing Aryl and Heteroaryl Groups: Enantioselective Synthesis of Bioactive Indole-Containing Triarylmethanes
}

Qiaolin YAN ${ }^{\dagger},{ }^{1}$ Meng Duan ${ }^{\dagger},{ }^{2,3}$ Cien Chen, ${ }^{4}$ Zhiqin Deng, ${ }^{5}$ Mandi Wu, ${ }^{4}$ Peiyuan Yu, ${ }^{3}$ Ming-liang He, ${ }^{* 4}$ Guangyu Zhu, ${ }^{* 5}$ K. N. Houk, ${ }^{* 2}$ Jianwei Sun*1

${ }^{1}$ Department of Chemistry, the Hong Kong University of Science and Technology, Clear Water Bay, Kowloon, Hong Kong SAR, China

${ }^{2}$ Department of Chemistry and Biochemistry, University of California, Los Angeles, California 90095, United States

${ }^{3}$ Department of Chemistry and Shenzhen Grubbs Institute, Guangdong Provincial Key Laboratory of Catalysis, Southern University of Science and Technology, Shenzhen, 518055, China

${ }^{4}$ Department of Biomedical Sciences, City University of Hong Kong, Kowloon Tong, Hong Kong SAR, China; and CityU Shenzhen Research Institute, Shenzhen, China

${ }^{5}$ Department of Chemistry, City University of Hong Kong, Kowloon Tong, Hong Kong SAR, China

These authors contributed equally to this work.

*e-mail: mlhe7788@gmail.com; guangzhu@cityu.edu.hk; houk@ucla.edu; sunjw@ust.hk

Abstract: Despite the enormous developments of asymmetric catalysis, the basis for asymmetric induction is largely limited to spatial interaction between substrate and catalyst. Consequently, asymmetric discrimination between two sterically similar groups remains a challenge. This is particularly formidable for enantiodifferentiation between aryl and heteroaryl groups without a directing group or electronic manipulation. Here we address this challenge by a robust organocatalytic system leading to excellent enantioselection between aryl and heteroaryl groups. With the versatile 2 -indole imine methide as platform, an excellent combination of a superb chiral phosphoric acid and the optimal hydride source provided 
efficient access to a range of highly enantioenriched indole-containing triarylmethanes. Control experiments and kinetic studies provided important insights into the mechanism. DFT calculations also indicated that, while hydrogen bonding is important for activation, the key interaction for discrimination of the two aryl groups is mainly $\pi$ - $\pi$ stacking. Preliminary biological studies also demonstrated the great potential of these triarylmethanes for anticancer and antiviral drug development.

Asymmetric catalysis has evolved arguably into the most powerful method for the synthesis of enantioenriched molecules. ${ }^{1}$ It features high efficiency and atom-economy in principle as compared to other approaches such as chiral resolution and auxiliary-based asymmetric synthesis, thereby enabling increasing applications in industrial synthesis. ${ }^{2}$ In the past few decades, a wide range of chiral catalytic systems with diverse activation modes have been developed. However, the fundamental basis for enantiocontrol remains essentially unchanged, i.e., spatial interaction between substrate and catalyst is still the key to success. ${ }^{1,2}$ For example, in the construction of a tetrahedral $\mathrm{C}\left(s p^{3}\right)$-chiral center from a prochiral $\mathrm{C}\left(s p^{2}\right)$-based planar substrate (e.g., carbocation, radical, carbonyl, olefin), a chiral catalyst typically provides enantiodifferentiation by blocking one face of the plane and directing the reaction partner $(\mathrm{Y})$ to approach towards the other face (Fig 1a). To achieve this, the catalyst must be able to effectively discriminate between the two substituents (R1 and R2) on the prochiral carbon. Obviously, the larger the difference of these two substituents is, the better enantioselectivity will be expected. Consequently, it has been well-established to achieve high enantioselectivity for cases bearing two sterically different groups (e.g., alkyl/aryl vs. H, large alkyl vs. small alkyl). In contrast, cases bearing two substituents of similar size remain challenging. ${ }^{1}$

1,1-Diarylmethinyl stereocenters is a widely prevalent structural motif in various natural products and biologically important molecules. ${ }^{3-4}$ Asymmetric addition to the 1,1-diaryl $\mathrm{C}=\mathrm{C}$ and $\mathrm{C}=\mathrm{X}(\mathrm{X}=$ heteroatom) bonds represents one of the most direct approaches for the construction of this unit. ${ }^{5-21}$ However, this requires effective discrimination between two (often) sterically similar aryl groups, which represents a notable challenge in asymmetric catalysis (Fig 1b). ${ }^{5}$ So far, success has mainly relied on the use of a directing group ${ }^{6-14}$ in one aryl group to allow catalyst recognition (e.g., by coordination) or electronic difference by incorporating electron-donating/withdrawing groups ${ }^{15,16}$. 
Notably, the effective enantiodifferentiation between aryl and heteroaryl groups still remains challenging, particularly in the absence of a directing group or electronic manipulation. ${ }^{17-21}$ Currently, successful differentiation between aryl and heteroaryl mostly involves a pyridine heterocycle due to its special coordinating ability. ${ }^{17-21}$ Moreover, despite the above-mentioned important progress, it is worth noting that almost all these examples relied on metal catalysis, and little success has been achieved by organocatalysis. In this context, here we describe an organocatalytic discrimination of non-directing aryl and heteroaryl groups with excellent enantioselectivity.

Indole imine methides (IIMs) have recently emerged as versatile intermediates for the asymmetric synthesis of enantioenriched indole derivatives, a family of useful units in medicinal chemistry. ${ }^{22-31}$ In particular, those with the methide motif adorned in the 2-position of indole are particularly useful to construct indole-fused polyheterocycles via asymmetric annulation processes, as pioneered by Shi and co-workers. ${ }^{22-28}$ However, the majority of these examples either involve straightforward discrimination (e.g., aryl vs. H) or do not involve asymmetric control in the 2-benzylic position (e.g, with two same substituents). ${ }^{22-31}$ In continuation of our efforts in this area, ${ }^{14,29-31}$ we envisioned that this type of intermediates would be a good platform to study the power of organocatalysis for the challenging discrimination between aryl and heteroaryl groups lacking a directing group (Scheme 1c). However, additional challenges should be expected since this intermediate II is likely generated as a $Z / E$ mixture, typically in equilibrium with carbocation $\mathbf{I}$. Therefore, the equilibrium should be made in synergy with the nucleophilic addition step to allow dynamic asymmetric control in order to achieve high enantioselectivity. 


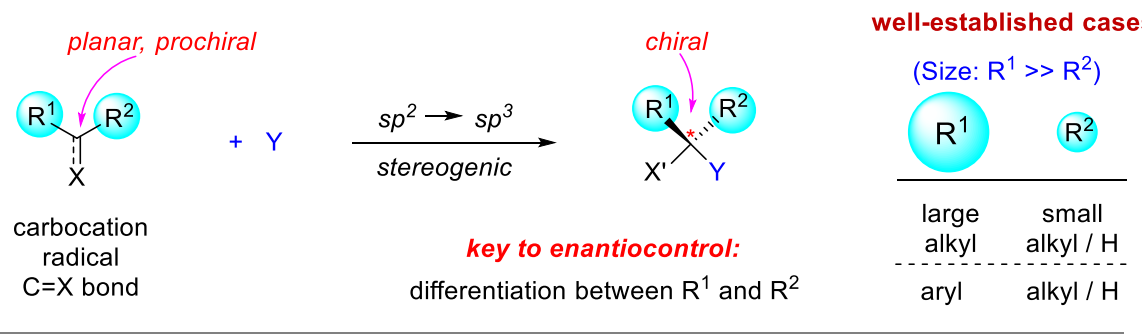

b

Discrimination between two aryl groups Typical Strategies: Aryl vs Aryl

Aryl vs Heteroaryl

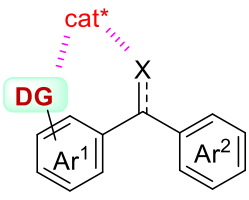

Directing group (DG)

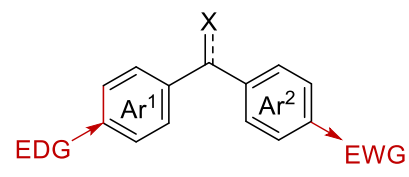

Electronic difference

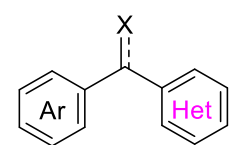

No directing group?

Rarely successful

Almost all the successful examples are based on metal catalysis so far
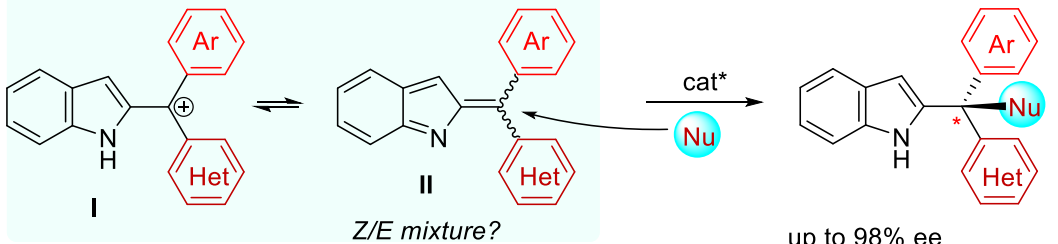

up to $98 \%$ ee

- Organocatalytic - No directing group - No electronic manipulation

Fig. 1. Introduction to asymmetric differentiation in $\mathbf{C}\left(s p^{2}\right)$-prochiral centers. a Asymmetric differentiation in prochiral planar substrates. b Challenges in discrimination between two aryl groups. c Our reaction design and the challenges.

\section{Results}

Reaction development. To test our hypothesis, we employed racemic tertiary alcohol 1a as the model precursor to the 2-indole imine methide intermediate. Notably, no directing group is incorporated in the two aryl groups (phenyl and thienyl) to be discriminated by catalyst. Despite the above-mentioned substantial challenges on this asymmetric control, considerable efforts were devoted to condition optimization and ultimately led to excellent reaction efficiency and enantiocontrol (Table 1). Specifically, among the broad range of chiral acid catalysts evaluated, the SPINOL-derived chiral phosphoric acid $\mathbf{C 1}$ was identified as the optimal catalyst. ${ }^{32-37}$ With benzothiazoline $\mathbf{2 a}$ as the hydride source, ${ }^{38,30}$ the asymmetric reduction proceeded smoothly to form indole-containing triarylmethane 3a under mild conditions in essentially quantitative yield and $95 \%$ 
ee (entry 1, Table 1). For comparison, other conditions typically led to inferior results. For example, other SPINOL-based chiral phosphoric acids gave lower enantioselectivity (entries 2-5). In particular, the previously well-known STRIP catalyst C2 resulted in only 16\% ee (entry 2). In addition, the catalyst chiral backbones were compared with the same 3,5-bis(trifluoromethyl)phenyl substituent in the 3,3'-positions (entries 3-5). While the SPINOL-based catalyst $\mathbf{C} 3$ gave $81 \%$ ee, in sharp contrast, the BINOL- and $\left[\mathrm{H}_{8}\right] \mathrm{BINOL}-$ derived analogues $\mathbf{A}$ and $\mathbf{B}$ did not show any asymmetric induction $(<2 \%$ ee). This result not only highlighted the superiority of the spirocyclic skeleton, but also corroborated the elusive stereocontrol in this case. Other hydride sources were also examined (entries 6-8). Benzothiazolines $\mathbf{2 b}$ and $\mathbf{2 c}$, bearing a different aryl substituent, led to drastically low chemoselectivity and enantioselectivity (entries 6-7). More surprisingly, Hantzsch ester $\mathbf{2 d}$, the previously well-established hydride source, ${ }^{39}$ gave drastically low enantiocontrol (entry 8). Other solvents than DCM did not provide a better result either (entries 9-11). The reaction was very sensitive to coordinating solvents, such as ether and ethyl acetate, which completely shut down the reaction, presumably due to competing binding with the acid catalyst. Decreasing the reaction temperature to $0{ }^{\circ} \mathrm{C}$ maintained high enantioselectivity, but moderately affected the reaction rate (entry 12). Finally, at a higher concentration, slightly lower enantioselectivity was observed (entry $13)$.

Table 1. Evaluation of reaction conditions. ${ }^{\mathrm{a}}$<smiles>N#Cc1c(C(O)(c2ccccc2)c2cccs2)[nH]c2ccccc12</smiles>

$$
\begin{aligned}
& +\quad \mathbf{2 a} \\
& \text { (1.1 equiv) }
\end{aligned}
$$

"standard conditions"

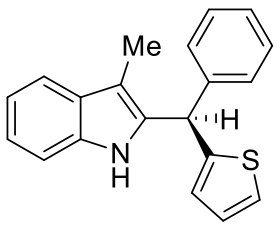

\begin{tabular}{|c|c|c|c|}
\hline Entry & $\begin{array}{l}\text { Deviation from the } \\
\text { "standard conditions" }\end{array}$ & Yield $(\%)^{b}$ & $\mathrm{ee}(\%)^{\mathrm{b}}$ \\
\hline 1 & none & $>95$ & 95 \\
\hline 2 & $(R)-\mathbf{C} 2$ instead of $(R)-\mathbf{C} \mathbf{1}$ & $>95$ & 16 \\
\hline 3 & $(R)-\mathbf{C 3}$ instead of $(R)-\mathbf{C 1}$ & $>95$ & 81 \\
\hline 4 & $(R)$-A instead of $(R)$-C1 & $>95$ & $<2$ \\
\hline 5 & $(R)$-B instead of $(R)$-C1 & $>95$ & $<2$ \\
\hline
\end{tabular}

$3 a$ 


\begin{tabular}{|c|c|c|c|}
\hline 6 & $\mathbf{2 b}$ instead of $\mathbf{2 a}$ & $11^{\mathrm{c}}$ & 80 \\
\hline 7 & $\mathbf{2 c}$ instead of $\mathbf{2 a}$ & $15^{\mathrm{c}}$ & 55 \\
\hline 8 & $\mathbf{2 d}$ instead of $\mathbf{2 a}$ & $78^{\mathrm{d}}$ & -9 \\
\hline 9 & $\mathrm{Et}_{2} \mathrm{O}$ as solvent & $<5^{\mathrm{e}}$ & - \\
\hline 10 & Toluene as solvent $^{\mathrm{a}}$ & 87 & 89 \\
\hline 11 & EtOAc as solvent $^{\mathrm{e}}$ & $<5^{\mathrm{e}}$ & - \\
\hline 12 & Run at $0{ }^{\circ} \mathrm{C}$ & $84^{\mathrm{d}}$ & 96 \\
\hline 13 & $c=0.2 \mathrm{M}$ & $>95$ & 93 \\
\hline
\end{tabular}<smiles>[R]c1cc(OP(=O)(O)Oc2c([R])cc3ccccc3c2-c2ccccc2)c2ccccc2c1</smiles>

$(R)-\mathbf{A}$ $\mathrm{R}=3,5-\left(\mathrm{CF}_{3}\right)_{2} \mathrm{C}_{6} \mathrm{H}_{3}$

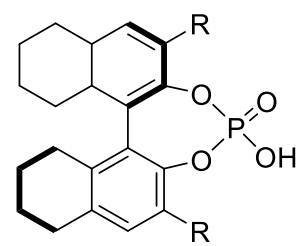

(R)-B $\mathrm{R}=3,5-\left(\mathrm{CF}_{3}\right)_{2} \mathrm{C}_{6} \mathrm{H}_{3}$

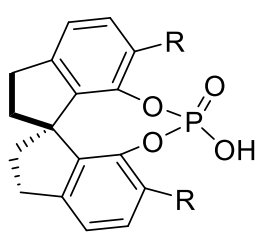

$(R)-\mathrm{C} 1: \mathrm{R}=3,5-\left({ }^{t} \mathrm{Bu}\right)_{2}-4-(\mathrm{OMe}) \mathrm{C}_{6} \mathrm{H}_{2}$ (R)-C2: $\mathrm{R}=2,4,6-\left({ }^{i} \mathrm{Pr}\right)_{3} \mathrm{C}_{6} \mathrm{H}_{2}$

(R)-C3: $\mathrm{R}=3,5-\left(\mathrm{CF}_{3}\right)_{2} \mathrm{C}_{6} \mathrm{H}_{3}$

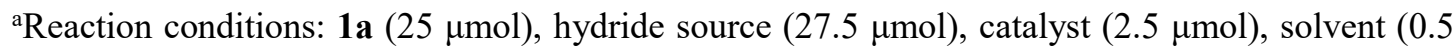
$\mathrm{mL})$. ${ }^{\mathrm{b}}$ Yield was determined by analysis of the ${ }^{1} \mathrm{H}$ NMR spectrum of the crude reaction mixture with $\mathrm{CH}_{2} \mathrm{Br}_{2}$ as internal standard. Ee was determined by HPLC analysis on a chiral stationary phase. ${ }^{\mathrm{c}} \mathrm{A}$ mixture of unidentifiable products was formed. ${ }^{\mathrm{d}} \mathrm{Clean}$ conversion. The starting material accounts for the remainder of the mass balance. ${ }^{e}$ Conversion $<5 \%$.

Substrates scope exploration. With the optimized conditions, we examined the reaction scope with variously substituted indole-derived tertiary alcohol substrates (Table 2). In general, this protocol provided efficient access to a wide range of highly enantioenriched indole-containing triarylmethanes. Substrates bearing electron-withdrawing and electron-donating groups at different positions were all suitable. The presence of a substituent at the 3-position of indole is not necessary (3f), although this position is nucleophilic and can potentially serve as a competitive intermolecular nucleophile. In addition to substitution at the 2-position of the thiophene ring in most cases, it is worth noting that substitution at the 3-position provided equally high enantioselectivity (3p). Finally, it is worth noting that, other than those thiophene-containing examples, the discrimination between benzene and furan ring is also possible, leading to good enantiocontrol (3q). In all these cases, no directing group to provide additional interaction (e.g., hydrogen bonding) with catalyst is needed in order to achieve high enantiocontrol. Steric perturbation on enantioselectivity by increasing the size of either of the two aryl groups (e.g., 31-m) was not obvious. Notably, the structure and absolution 
configuration of products $\mathbf{3 a}$ and $\mathbf{3 p}$ were confirmed by X-ray crystallography.

Table 2. Reaction scope. ${ }^{\mathrm{a}}$
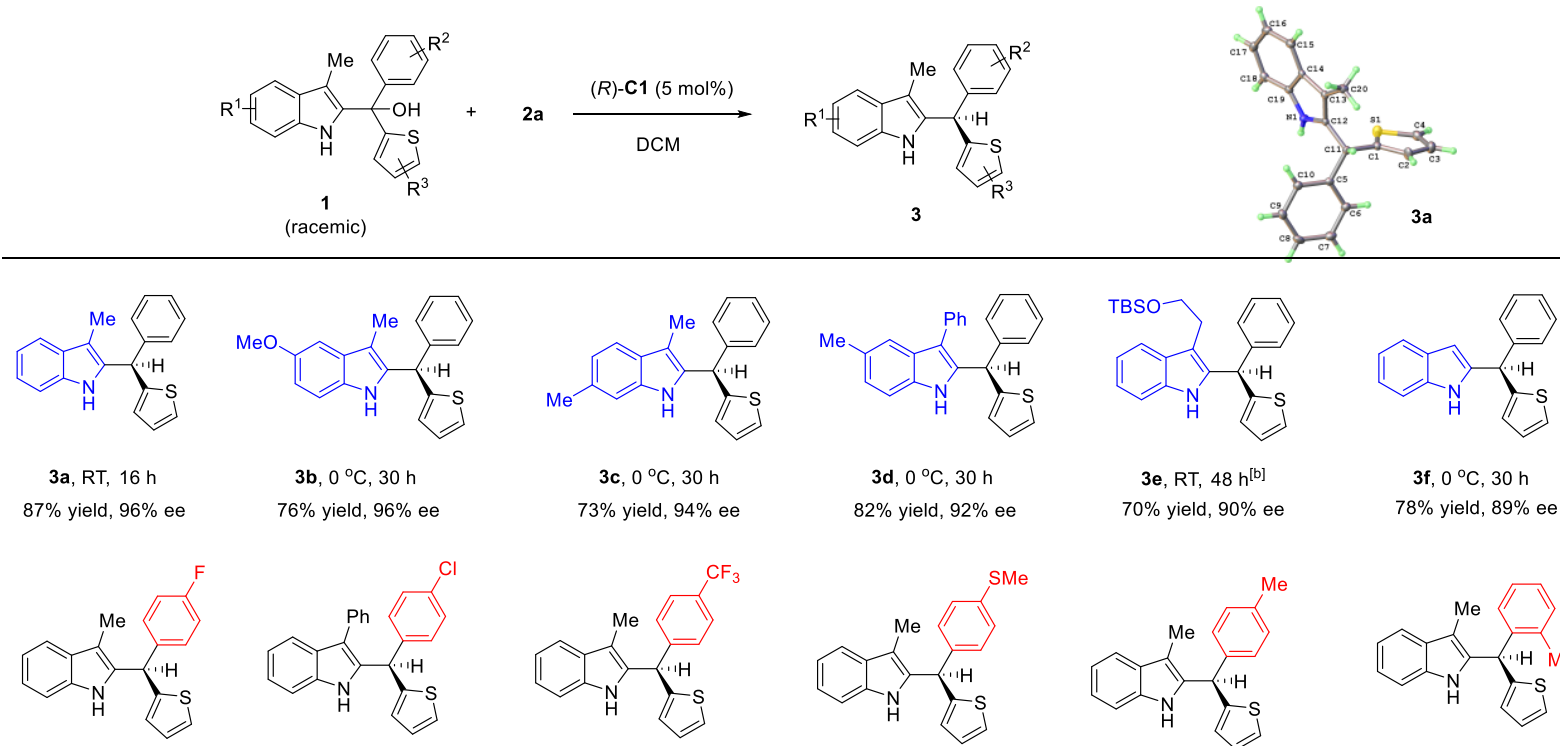

$$
76 \% \text { yield, } 96 \% \text { ee }
$$
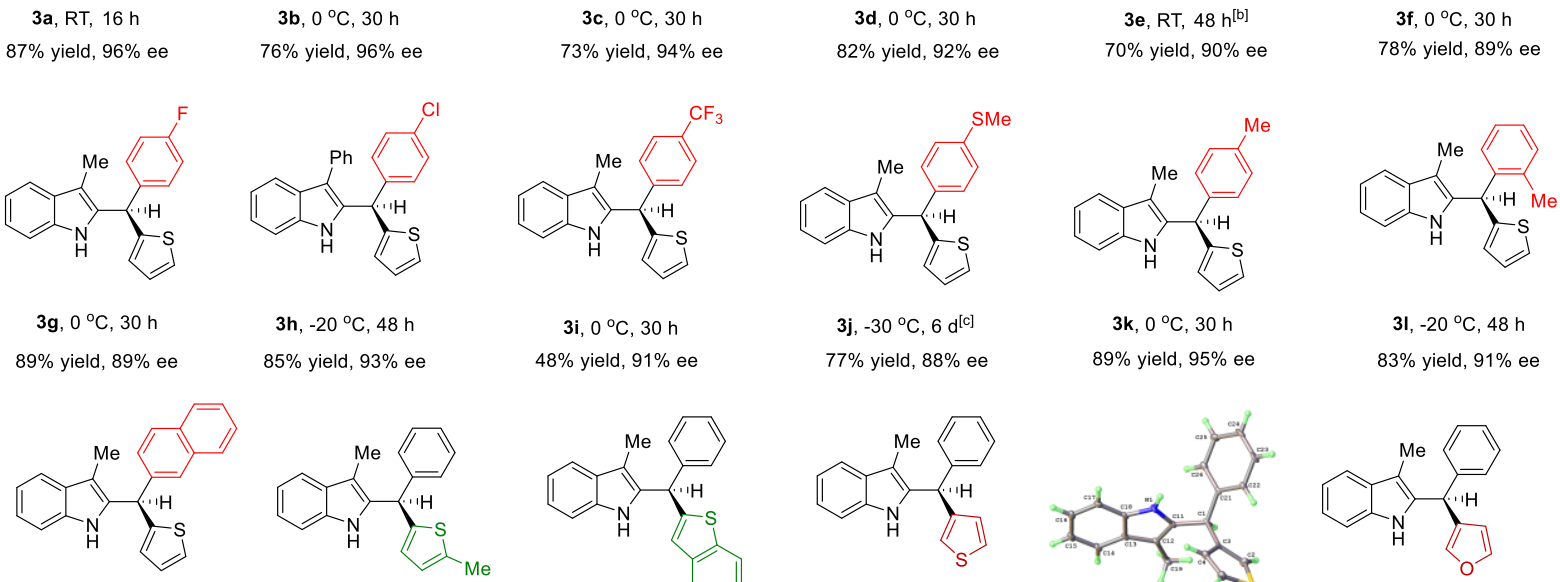

$$
\begin{gathered}
3 \mathrm{~h},-20^{\circ} \mathrm{C}, 48 \mathrm{~h} \\
85 \% \text { yield, } 93 \% \text { ee }
\end{gathered}
$$

3i, $0{ }^{\circ} \mathrm{C}, 30 \mathrm{~h}$ $48 \%$ yield, $91 \%$ ee

$$
3 j,-30{ }^{\circ} \mathrm{C}, 6 \mathrm{~d}^{[\mathrm{c}]}
$$

3k, $0{ }^{\circ} \mathrm{C}, 30 \mathrm{~h}$ $89 \%$ yield, $95 \%$ ee 31, $-20^{\circ} \mathrm{C}, 48 \mathrm{~h}$ $77 \%$ yield, $88 \%$ ee
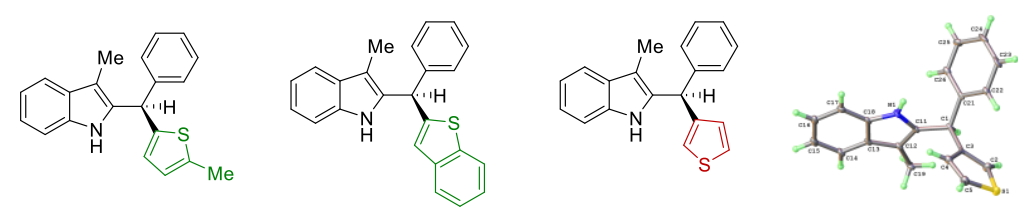

$83 \%$ yield, $91 \%$ ee

$3 \mathrm{~m}, 0^{\circ} \mathrm{C}, 30 \mathrm{~h}$

3n, $0{ }^{\circ} \mathrm{C}, 30 \mathrm{~h}$

3o, $0^{\circ} \mathrm{C}, 72 \mathrm{~h}$

$3 p, 0{ }^{\circ} \mathrm{C}, 41 \mathrm{~h}$

$3 p$

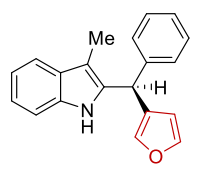

$85 \%$ yield, $92 \%$ ee

3q, RT, $12 \mathrm{~h}$ $44 \%$ yield, $84 \%$ ee

${ }^{a}$ Reaction scale: 1 (0.4 mmol, 2 (0.44 mmol), $(R)-C 1$ (5 mol\%), DCM (8.0 mL). ${ }^{b}$ Run with $10 \mathrm{~mol} \%$ of catalyst.

The robustness of this protocol was further examined by stoichiometric adulteration of various additives bearing different functional groups ${ }^{40}$ As shown in Table 3, in most cases, the excellent chemical efficiency and enantioselectivity were not obviously affected by the additives in the standard reaction of 1a and 2a. Many of these additives contain highly polar and reactive functionalities that are typical strong hydrogen-bonding partners, such as primary amine, thiol, alcohol, carbonyl, sulfone, and boronic acid. This is particularly remarkable in view of the fact that hydrogen bonding is a key catalyst-substrate interaction in the process. Notably, from a different point of view, the little influence on enantiocontrol by polar additives might also imply that it is not hydrogen bonding, but other interactions such as $\pi-\pi$ stacking, that provide the basis for asymmetric 
discrimination (vide infra). Nevertheless, these results clearly illustrated the excellent functional group tolerance as well as the robust enantiodifferentiation ability of this mild but powerful catalytic system.

Table 3. Compatibility of external functional groups. ${ }^{a}$

\begin{tabular}{|c|c|c|c|c|c|}
\hline & & & ard conditions & 2 & \\
\hline & & & $\mathrm{A}, \mathrm{rt}, 12 \mathrm{~h}$ & & \\
\hline Entry & Additive & $\begin{array}{c}\text { Recovered } \\
\text { additive (\%) }\end{array}$ & $\begin{array}{c}\text { Conversion } \\
\text { of } \mathbf{1 a}(\%)\end{array}$ & $\begin{array}{l}\text { Yield of } \\
\mathbf{3 a}(\%)\end{array}$ & $\begin{array}{c}\text { Ee of } \mathbf{3 a} \\
(\%)\end{array}$ \\
\hline 1 & & 90 & 100 & 95 & 91 \\
\hline 2 & & 82 & 70 & 49 & 94 \\
\hline 3 & & 95 & 100 & 88 & 96 \\
\hline 4 & & 85 & 100 & 99 & 94 \\
\hline 5 & & 95 & 100 & 87 & 94 \\
\hline 6 & $\mathrm{Ph}_{3} \mathrm{CSH}$ & 66 & 100 & 83 & 89 \\
\hline 7 & & 61 & 100 & 77 & 87 \\
\hline 8 & & 67 & 100 & 73 & 94 \\
\hline 9 & & 78 & 100 & 64 & 90 \\
\hline 10 & & 83 & 100 & 75 & 94 \\
\hline 11 & $\mathrm{PPh}_{3}$ & 80 & 100 & 95 & 94 \\
\hline 12 & & 67 & 100 & 57 & 94 \\
\hline
\end{tabular}




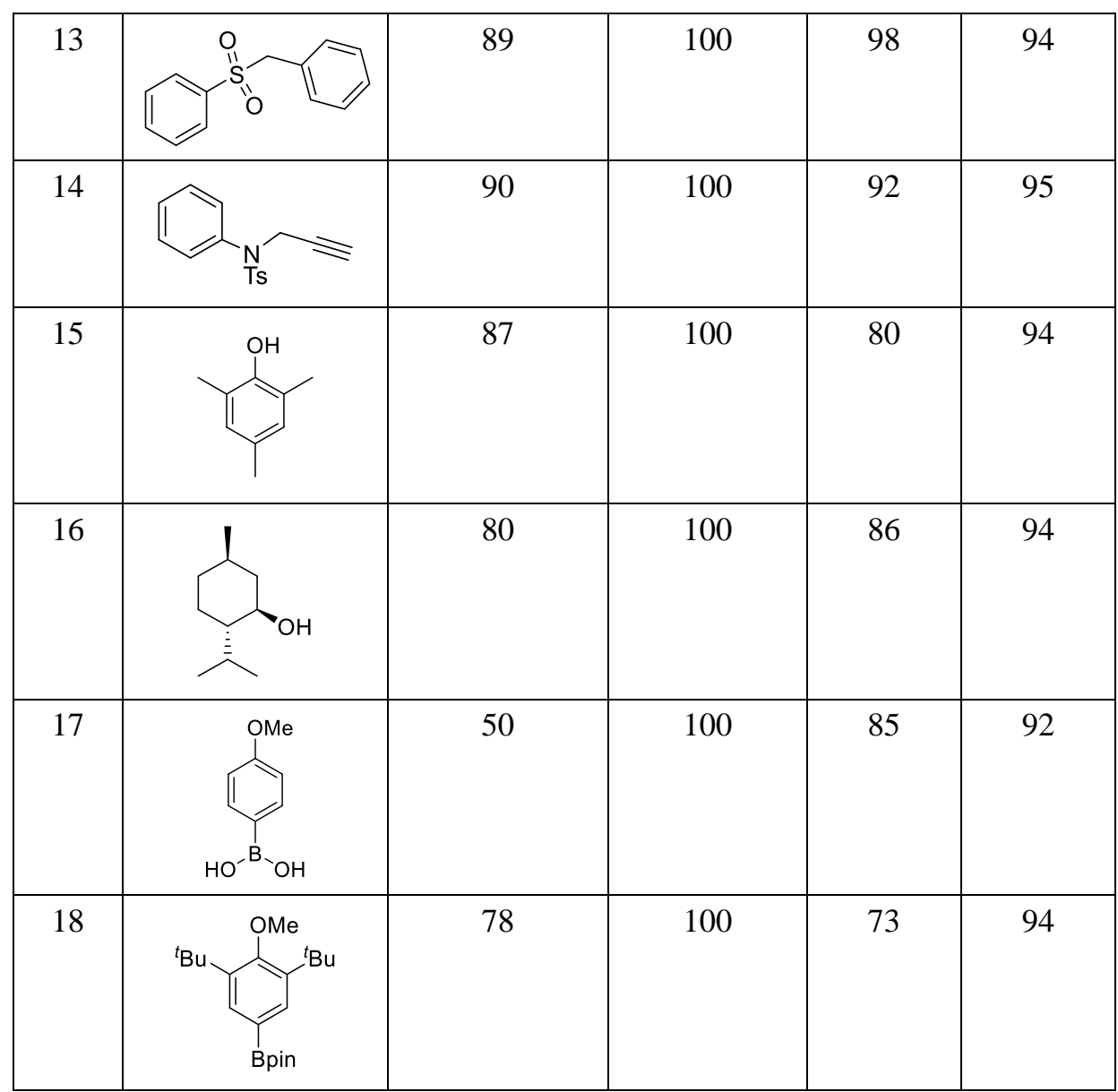

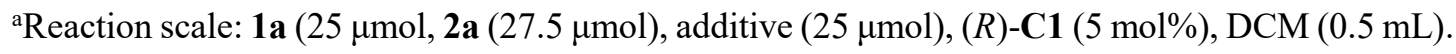
Yield was determined by analysis of the ${ }^{1} \mathrm{H}$ NMR spectrum of the crude reaction mixture with $\mathrm{CH}_{2} \mathrm{Br}_{2}$ as internal standard. Ee was determined by HPLC analysis on a chiral stationary phase.

Mechanistic studies. A possible mechanism is proposed in Fig 2. We believe that this reaction begins with acid-catalyzed dehydration to from indolyl cation IM, paired with a phosphate counter anion. This ion pair might be in equilibrium (or pseudo resonance) with the activated indole imine methide form IM'. Subsequently, the hydride source approaches the benzylic carbon to deliver the product 3 . 

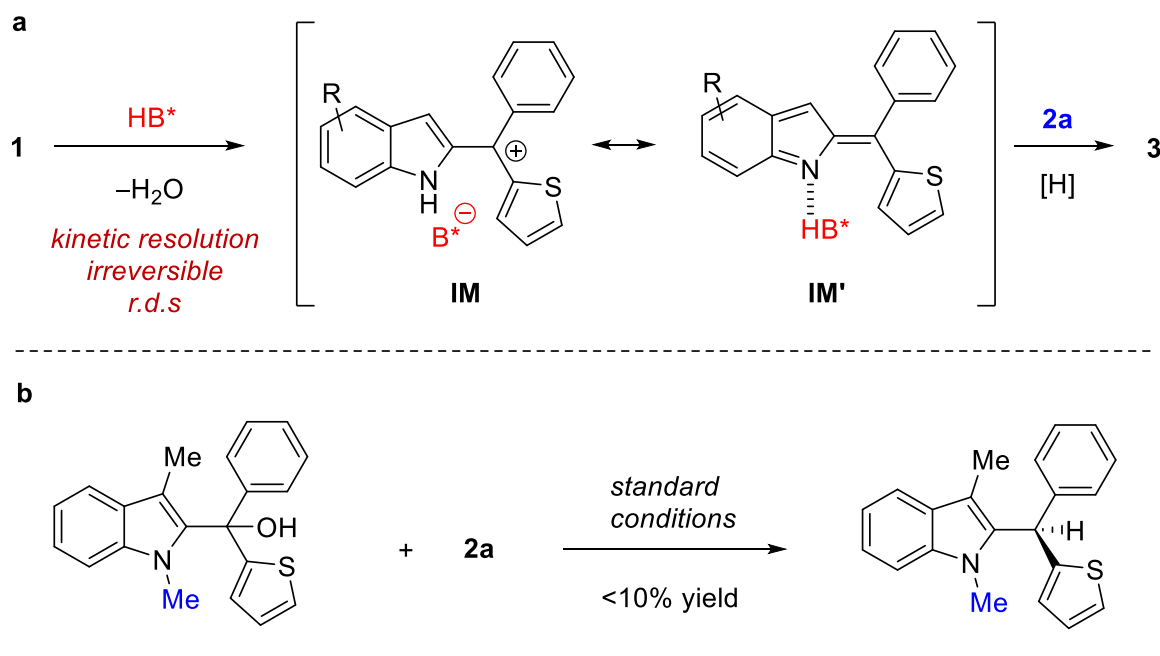

$1 a^{\prime}$

$3 a^{\prime}$

Fig. 2. Proposed mechanism and a control experiment. a Proposed mechanism b Control experiments with $N$-substituted substrate.

We have carried out a series of control experiments. First of all, under the standard conditions, the reaction with $N$-methylated substrate 1a' did not proceed to form the desired product 3a' (Scheme $3 b)$. This result suggests that the free $\mathrm{N}-\mathrm{H}$ motif in the indole moiety is essential to the observed reactivity, which is consistent with the intermediacy of 2-indole imine methide IM', as this intermediate cannot be formed from 1a'. Next, the enantiomeric excess (ee) values of substrate and product were both monitored during the reaction progress (Fig 3a). The product ee remained constant $(95 \%$ ee) during the entire reaction, but substrate ee gradually increased over time. This enantioconvergent feature agrees with the initial formation of an achiral 2-indole imine methide intermediate followed by stereodefined asymmetric addition of nucleophile. The observation of substrate enantioenrichment is indicative of kinetic resolution during the first step, which is likely irreversible. Taken together, a direct $\mathrm{S}_{\mathrm{N}} 2$ mechanism could be excluded. Furthermore, this reaction did not exhibit non-linear effects, suggesting that the enantiodetermining transition state likely involves only one catalyst molecule. Finally, kinetic studies indicated that this reaction exhibits zeroth order in nucleophile and first order in catalyst, which further confirmed that the first step is rate-determining and irreversible. 

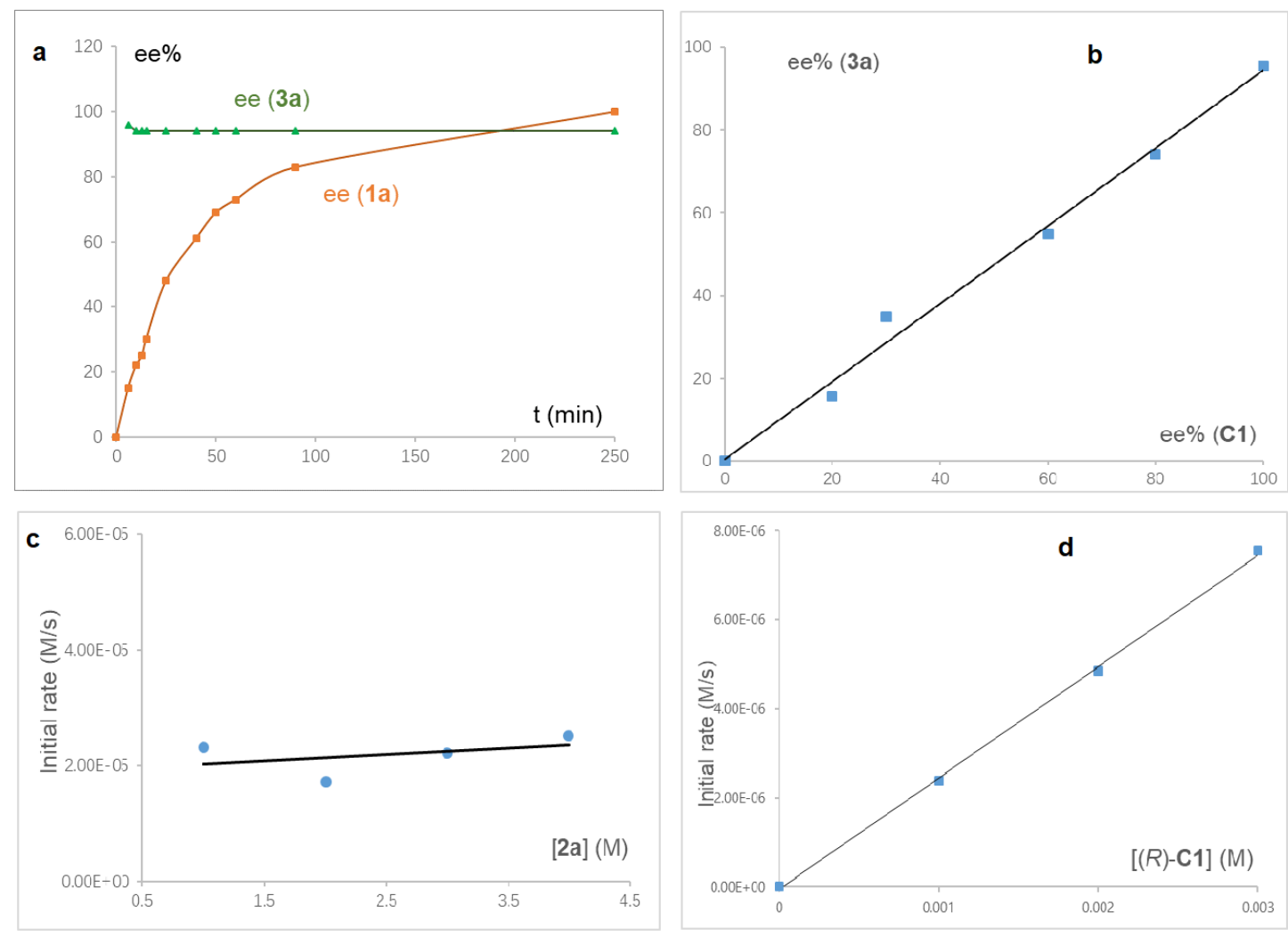

Fig. 3. Mechanistic studies. a Time-dependence of substrate and product ee values. b Absence of non-linear effects. $\mathbf{c}$ Zeroth order in nucleophile. $\mathbf{d}$ First order in catalyst.

Density functional theory studies. The rate-determining step of this reaction is the chiral phosphoric acid-catalyzed dehydration of tertiary alcohols 1a to obtain the indolyl cation IM. However, the stereo-determining step for this reaction is the nucleophilic addition of the hydride 2a on the indolyl cation IM. To evaluate the origins of stereoselectivity, density functional theory (DFT) calculations were performed. We first explored the stereo-determining transition structures (Fig. 4). Geometry optimizations were conducted at B3LYP-D3(BJ) functional with the def2svp basis set in the Gaussian 16 package. ${ }^{41-43}$ Single-point energies and solvent effects in dichloromethane were calculated with the CPCM solvation model at the M06-2X/def2tzvpp level of theory. ${ }^{44-47}$ Conformational searches were conducted using the CREST conformer-rotamer ensemble sampling tool version 2.10.2 with xtb version 6.3.3. ${ }^{48-52}$ Based on the results of conformational searches, the lowest energy conformers are depicted in Figure 2. The computed transition state TS-R delivering the preferred R-product is $3.6 \mathrm{kcal} / \mathrm{mol}$ lower than that of TS-S generating the S-product, in good accord with the $96 \%$ ee observed experimentally. 

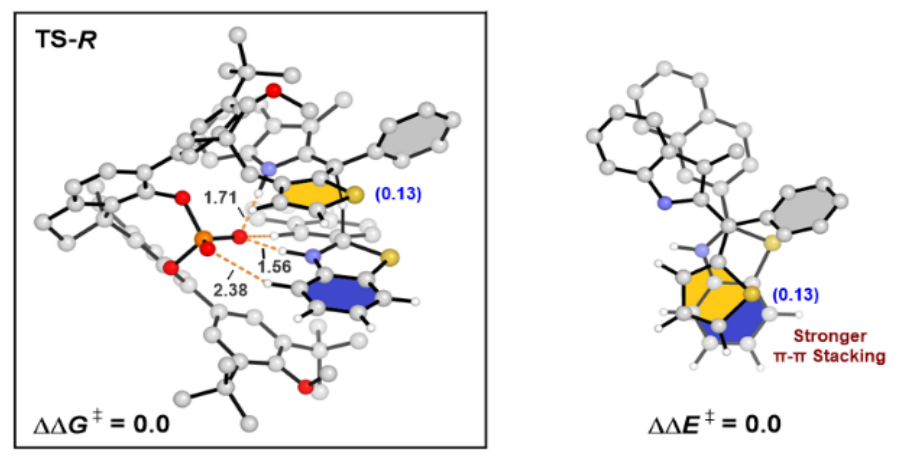

$\Delta \Lambda E^{\ddagger}=0.0$

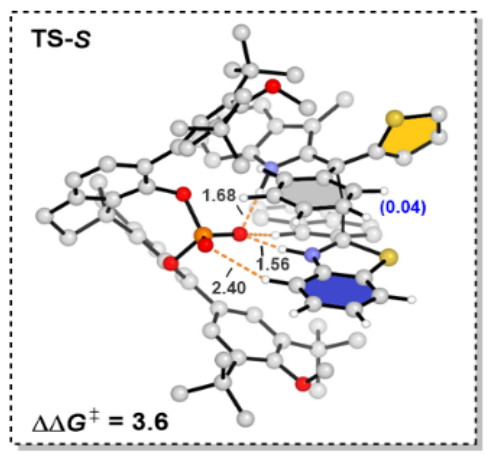

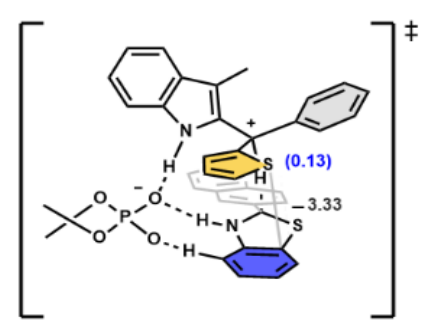

TS- $R$

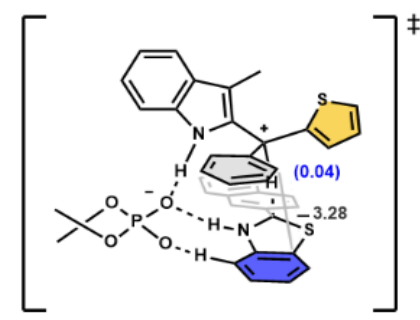

TS-S

Fig. 4. DFT-optimized stereo-determining transition structures. The distances are given in Ångstroms, and energies are given in kcal/mol. Coloured rings: grey, phenyl; yellow, thienyl; blue, benzo group on the benzothiazoline. Numbers in parentheses are the total Hirshfeld charges on the aryl groups.

To gain further insights into the factors that impact the enantioselectivity, the geometries of transition states TS-R and TS-S were compared. No obvious steric clashes and hydrogen-bonding interaction difference between the catalyst and substrates are detected in these two competing transition states. Computational studies of the total Hirshfeld charges on the aryl groups show that the key interaction for discrimination of the two aryl groups is mainly $\pi-\pi$ stacking. Thienyl is a better donor than phenyl so it donates more electrons to the $\mathrm{C}+$. In major TS-R, the electrondeficient thienyl $(0.13 \mathrm{e})$ is in closer contact with the electron-rich benzo ring of the benzothiazoline. By contrast, in minor TS-S, the phenyl group $(0.04 \mathrm{e})$ forms a slip-stacked configuration with the benzo group on the hydride. As a result, the stronger $\pi-\pi$ stacking stabilizes TS-R more than the weaker $\pi-\pi$ stacking stabilizes TS-S. This conclusion rather than some interaction of transition state with catalyst was tested by calculations on the fixed transition state formed by removing the catalyst, Single-point $\Delta \Delta \mathrm{E} \ddagger$ without optimization show a $2.5 \mathrm{kcal} / \mathrm{mol}$ advantage for the stronger attractive $\pi-\pi$ stacking in TS-R. This is the significant contribution to the $3.6 \mathrm{kcal} / \mathrm{mol}$ preference for the 
formation of R-product. Therefore, attractive $\pi-\pi$ stacking plays a major role in the selectivity.

Biological Studies. To investigate the potential anticancer activity of the enantioenriched indolecontaining triarylmethanes, we examined the cytotoxicity of the representative product $\mathbf{3 d}$ towards human cervical adenocarcinoma (HeLa), ovarian carcinoma (A2780), breast adenocarcinoma (MCF-7), colorectal carcinoma (HCT116), and lung carcinoma (A549) cells. A widely used anticancer drug, doxorubicin, was used as the control. As shown in Table 4, 3d exhibited significant cytotoxicity, with the half-maximal inhibitory concentration (IC50) values ranging from 5.6 to 18.2 $\mu \mathrm{M}$. A549 lung carcinoma cells were found to be the most sensitive cell line towards $\mathbf{3 d}$, and a normal cell line from the same origin (MRC-5 lung fibroblasts) was used to evaluate the cancer cell selectivity of 3d. The IC50 value in MRC-5 cells is $27.1 \mu \mathrm{M}$, corresponding to a selectivity index (SI; cytotoxicity in the normal cells / cytotoxicity in the cancer cells) of 4.8; whereas the SI value for doxorubicin is only 2.6. These preliminary results suggest that this class of compounds have promising potential for further development as anticancer drug candidates.

Table 4. The cytotoxicity of $\mathbf{3 d}$ in various human cell lines.

\begin{tabular}{|c|c|c|c|c|c|c|}
\hline \multirow{2}{*}{ Cell line } & \multicolumn{5}{|c|}{ IC $_{50}$ value $(\mu \mathrm{M})^{\mathrm{a}}$} \\
\cline { 2 - 7 } & HeLa & MCF-7 & A2780 & A549 & HCT116 & MRC-5 \\
\hline Doxorubicin & $1.4 \pm 0.4$ & $0.55 \pm 0.11$ & $0.36 \pm 0.07$ & $0.28 \pm 0.06$ & $1.4 \pm 0.3$ & $0.72 \pm 0.14$ \\
\hline 3d & $18.2 \pm 2.9$ & $15.3 \pm 2.3$ & $8.9 \pm 1.7$ & $5.6 \pm 0.9$ & $9.7 \pm 1.3$ & $27.1 \pm 3.4$ \\
\hline
\end{tabular}

${ }^{a}$ The half inhibitory concentration (IC50) values were determined by MTT assay in $72 \mathrm{~h}$. The error bars were obtained as the standard deviation from the mean value based on three independent experiments.

We also tested the antiviral activity of another representative product 3a with enterovirus A71 (EVA71) using Rhabdomyosarcoma (RD) cell line. The cytopathic effect (CPE) and intracellular viral RNA level were measured to reflect the antiviral effects. Indeed, a strong CPE was observed after EV-A71 infection at the multiplicity of infection (MOI) of 0.01 for 36 hours. The morphology of RD cells changed from flat to unhealthy round and death. As shown in Fig. 5a, the CPE induced by EV-A71 infection was significantly reduced upon treatment with 3a. Moreover, the intracellular viral RNA level was decreased by $80-90 \%$ after treating with 3a at the concentration of 5-10 $\mu \mathrm{M}$ 
compared with untreated EV-A71 infected cells (Fig. 5b). The strong antiviral effect of 3a was also confirmed by viral titration. The virus titer was decreased by 35 folds upon treatment with 3a (Fig. 5c). Moreover, this compound showed low cytotoxicity according to the MTT assay (Table 5), thus indicating a high selectivity index and suggesting a great potential of such molecules for antiviral drug development.

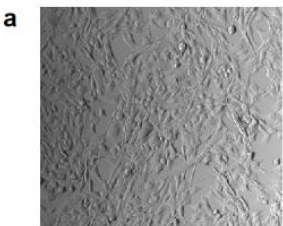

RD MOCK

b

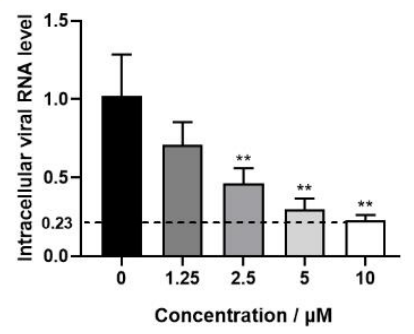

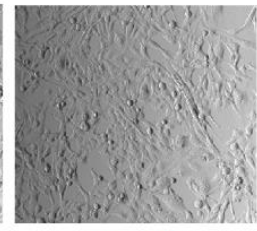

$3 a(5 \mu M)$

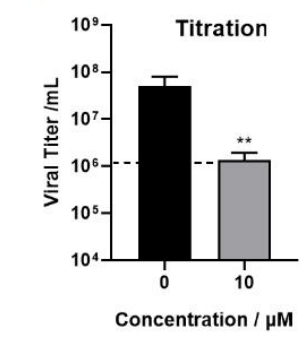

Fig. 5. The antiviral effects of 3a shown by CPE assay and intracellular viral RNA level. a RD cells were first treated with compounds at different concentration and then infected with EV-A71 at MOI of 0.01 after 2 hours. Cell morphology was observed $36 \mathrm{~h}$ post-infection. b Relative intracellular EV-A71 genome RNA level was determined by RT-qPCR. $\mathbf{c}$ The EV-A71 viral titer in the supernatant was measured by TCID50 assay. Data are represented as mean $\pm \mathrm{SD}(\mathrm{n}=3)$. ${ }^{* *} \mathrm{p}<$ 0.01, compared with no infected group.

Table 5. Cytotoxicity concentration $\left(\mathrm{CC}_{50}\right)$ and antiviral activity $\mathrm{IC}_{50}{ }^{\mathrm{a}}$

\begin{tabular}{|c|c|c|c|}
\hline Compound & $\mathrm{CC}_{50}(\mu \mathrm{M})$ & $\mathrm{IC}_{50}(\mu \mathrm{M})$ & Selectivity index \\
\hline 3a & 55.46 & 2.27 & 24.43 \\
\hline
\end{tabular}

${ }^{\text {a }} \mathrm{CC}_{50,} 50 \%$ cytotoxic concentration tested by the viability assay with no viral infection. $\mathrm{IC}_{50}$, viral RNA copies deceased 50\% compared with the control group (without compound treatment) in the secreted virions. A selectivity index $\left(\mathrm{CC}_{50} / \mathrm{IC}_{50}\right)>10$ is assumed as a potential candidate for further research analysis. 


\section{Conclusion}

Despite the longstanding challenge on asymmetric discrimination between two sterically similar diaryl groups and the dominant role of metal catalysis in the limited previous studies, here we have demonstrated a rare organocatalytic example with excellent efficiency and enantiocontrol. The versatile 2-indole imine methide bearing aryl and heteroaryl groups without a directing group was used as a platform for this study. The combined use of a superb chiral phosphoric acid catalyst and a benzothioazoline hydride source is critically important to the success. This protocol provided efficient access to a wide range of highly enantioenriched indole-containing triarylmethanes from the corresponding racemic tertiary alcohols. Mechanistic experiments, including control reactions and kinetic studies, provided important insights into the mechanism, which involves initial ratedetermining dehydration (with concomitant substrate kinetic resolution) and subsequent enantioconvergent nucleophilic addition. Further DFT studies suggested that it is $\pi-\pi$ stacking, but not hydrogen bonding, that provides the key interaction for asymmetric discrimination between the benzene and thiophene rings. This is also consistent with the robust enantiocontrol in the presence of various polar functional groups that are likely hydrogen-bond destroyers. Preliminary biological studies also demonstrated the great potential of these triarylmethanes for anticancer and antiviral drug development.

\section{Methods}

\section{General procedure for the synthesis of enantioenriched triarylmethanes 3 .}

At $0{ }^{\circ} \mathrm{C}$, to an oven-dried $30-\mathrm{mL}$ vial were added the tertiary alcohol $\mathbf{1}(0.40 \mathrm{mmol})$, the hydride source 2a (115.9 mg, $0.44 \mathrm{mmol}),(R)-\mathbf{C 1}(15.04 \mathrm{mg}, 0.02 \mathrm{mmol}, 5.0 \mathrm{~mol} \%)$ and DCM (8.0 mL). The mixture was stirred at the same temperature for $30 \mathrm{~h}$ and concentrated under reduced pressure. The residue was purified by silica gel flash chromatography to afford the desired product.

\section{References}

1. Carreira, E. M. \& Yamamoto, H. Comprehensive Chirality, 1st Ed., Elsevier Science (2012).

2. Blaser, H. U. \& Federsel, H.-J. Asymmetric Catalysis on Industrial Scale: Challenges, Approaches, and Solutions, Wiley-VCH (2010). 
3. Ameen, D. \& Snape, T. J. Chiral 1,1-diaryl compounds as important pharmacophores. Med. Chem. Commun. 4, 893-907 (2013).

4. Mondal, S., Roy, D. \& Panda, G. Overview on the recent strategies for the enantioselective synthesis of 1,1-diarylalkanes, triarylmethanes and related molecules containing the diarylmethine stereocenter. ChemCatChem 10, 1941-1967 (2018).

5. Besset, T., Gramage-Doria, R. \& Reek, J. N. H. Remotely controlled iridium-catalyzed asymmetric hydrogenation of terminal 1,1-diaryl alkenes. Angew. Chem. Int. Ed. 52, 8795-8797 (2013).

6. Mazuela, J. et al. Iridium phosphite-oxazoline catalysts for the highly enantioselective hydrogenation of terminal alkenes. J. Am. Chem. Soc., 131, 12344-12353 (2009).

7. Touge, T., Nara, H., Fujiwhara, M., Kayaki, Y. \& Ikariya, T. Efficient access to chiral benzhydrols via asymmetric transfer hydrogenation of unsymmetrical benzophenones with bifunctional oxo-tethered ruthenium catalysts. J. Am. Chem. Soc. 138, 10084-10087 (2016).

8. Ohkuma, T., Koizumi, M., Ikehira, H., Yokozawa \& T., Noyori, R. Selective hydrogenation of benzophenones to benzhydrols. Asymmetric synthesis of unsymmetrical diarylmethanols. Org. Lett. 2, 659-662 (2000).

9. Wang, H. et al. Chiral electron-rich PNP Ligand with a phospholane motif: structural features and application in asymmetric hydrogenation. Org. Lett. 22, 8796-8801 (2020).

10. Song, S., Zhu, S.-F., Yu, Y.-B. \& Zhou, Q.-L. Carboxy-directed asymmetric hydrogenation of 1,1-diarylethenes and 1,1-dialkylethenes. Angew. Chem. Int. Ed. 52, 1556-1559 (2013).

11. Wang, X. et al. Highly enantioselective hydrogenation of styrenes directed by 2 '-hydroxyl groups. Org. Lett. 13, 1881-1883 (2011).

12. Liu, W., Guo, J., Xing, S. \& Lu, Z. Highly enantioselective cobalt-catalyzed hydroboration of diaryl Ketones. Org. Lett. 22, 2532-2536 (2020).

13. Zheng, Y., Clarkson, G. J. \& Wills, M. Asymmetric transfer hydrogenation of o-hydroxyphenyl ketones: utilizing directing effects that optimize the asymmetric synthesis of challenging alcohols. Org. Lett. 22, 3717-3721 (2020).

14. Li, X. et al. Catalytic enantioselective synthesis of chiral tetraarylmethanes. Nat. Catal. 3, $1010-1019$ (2020).

15. Yang, L.-L. et al. Enantioselective diarylcarbene insertion into $\mathrm{Si}-\mathrm{H}$ bonds induced by 
electronic properties of the carbenes. J. Am. Chem. Soc. 142, 12394-12399 (2020).

16. Lee, M., Ren, Z., Musaev, D. G. \& Davies, H. M. L. Rhodium-stabilized diarylcarbenes behaving as donor/acceptor carbenes. ACS Catal. 10, 6240-6247 (2020).

17. Yang, H., Huo, N., Yang, P., Pei, H., Lv, H. \& Zhang X. Rhodium catalyzed asymmetric hydrogenation of 2-pyridine ketones. Org. Lett. 17, 4144-4147 (2015).

18. Wang, B., Zhou, H., Lu, G., Liu, Q. \& Jiang, X. Bifunctional oxo-tethered ruthenium complex catalyzed asymmetric transfer hydrogenation of aryl N-heteroaryl ketones. Org. Lett. 19, 2094-2097 (2017).

19. Levedev, Y., Polishchuk, I., Maity, B., Guerreiro, M. D. V., Cavallo, L. \& Rueping, M. Asymmetric hydroboration of heteroaryl ketones by aluminum catalysis. J. Am. Chem. Soc. 141, 19415-19423 (2019).

20. Chen, F. et al. Chirality-economy catalysis: asymmetric transfer hydrogenation of ketones by Ru-catalysts of minimal stereogenicity. ACS Catal. 9, 5562-5566 (2019).

21. He, D., Xu, X., Lu, Y., Zhou, M.-J. \& Xing, X. Asymmetric Transfer Hydrogenation of densely functionalized diheteroaryl and diaryl ketones by a Ru-catalyst of minimal stereogenicity. Org. Lett. 22, 8458-8463 (2020).

22. Wang, L., Chen, Y. \& Xiao, J. Alkylideneindoleninium ions and alkylideneindolenines: key intermediates for the asymmetric synthesis of 3-indolyl derivatives. Asian J. Org. Chem. 3, $1036-1052$ (2014)

23. Zhang, Y.-C., Jiang, F. \& Shi, F. Organocatalytic asymmetric synthesis of indole-based chiral heterocycles: strategies, reactions, and outreach. Acc. Chem. Res. 53, 425-446 (2020).

24. Kikuchi, J. \& Terada, M. Enantioconvergent substitution reactions of racemic electrophiles by organocatalysis. Chem. Eur. J. 27, 10215-10225 (2021).

25. Qi, S., Liu, C.-Y., Ding, J.-Y. \& Han, F.-S. Chiral phosphoramide-catalyzed enantioselective synthesis of 2,3'-diindolylarylmethanes from indol-2-yl carbinols and indoles. Chem. Commun. 50, 8605-8608 (2014).

26. Sun, X.-X., Zhang, H.-H., Li, G.-H., He, Y.-Y. \& Shi, F. Catalytic enantioselective and regioselective [3+3] cycloadditions using 2-indolylmethanols as $3 \mathrm{C}$ building blocks. Chem. Eur. J. 22, 17526-17532 (2016).

27. Zhang, H.-H., Wang, C.-S., Li, C., Mei, G.-J., Li, Y. \& Shi, F. Design and enantioselective 
construction of axially chiral naphthyl-indole skeletons. Angew. Chem. Int. Ed. 56, 116-121 (2017).

28. Goricke, F. \& Schneider, C. Palladium-catalyzed, enantioselective $(3+2)$-cycloannulation of $\beta$-keto esters with alkylidene $2 \mathrm{H}$-indoles toward complex indole-based heterocycles. Org. Lett. 22, 6101-6106 (2020).

29. Li, X. \& Sun, J. Organocatalytic enantioselective synthesis of chiral allenes: remote asymmetric 1,8-addition of indole imine methides. Angew. Chem. Int. Ed. 59, 17049-17054 (2020).

30. Li, X., Duan, M., Yu, P., Houk, K. N. \& Sun, J. Organocatalytic enantioselective dearomatization of thiophenes by 1,10-conjugate addition of indole imine methides. Nat. Commun. 12, 4881 (2021).

31. Wang, Z. et al. Organocatalytic asymmetric synthesis of 1,1-diarylethanes by transfer hydrogenation. J. Am. Chem. Soc. 137, 383-389 (2015).

32. Akiyama, T., Itoh, J., Yokota \& K., Fuchibe, K. Enantioselective mannich-type reaction catalyzed by a chiral brønsted acid. Angew. Chem. Int. Ed. 43, 1566-1568 (2004).

33. Uraguchi, D. \& Terada, M. Chiral brønsted acid-catalyzed direct mannich reactions via electrophilic activation. J. Am. Chem. Soc. 126, 5356-5357 (2004).

34. Nakashima, D. \& Yamamoto, H. Design of chiral N-triflyl phosphoramide as a strong chiral brønsted acid and its application to asymmetric Diels-Alder reaction. J. Am. Chem. Soc. 128, 9626-9627 (2006).

35. Parmar, D., Sugiono, E., Raja \& S., Rueping, M. Complete field guide to asymmetric BINOLphosphate derived brønsted acid and metal catalysis: history and classification by mode of activation; brønsted acidity, hydrogen bonding, ion pairing, and metal phosphates. Chem. Rev. 114, 9047-9153 (2014)

36. Akiyama, T. \& Mori, K. Stronger brønsted acids: recent progress. Chem. Rev. 115, 9277-9306 (2015).

37. James, T., van Gemmeren \& M., List, B. Development and applications of disulfonimides in enantioselective organocatalysis. Chem. Rev. 115, 9388-9409 (2015).

38. Zhu, C., Saito, K., Yamanaka, M. \& Akiyama, T. Benzothiazoline: versatile hydrogen donor for organocatalytic transfer hydrogenation. Acc. Chem. Res. 48, 388-398 (2015). 
39. You, S.-L. Recent developments in asymmetric transfer hydrogenation with Hantzsch esters: a biomimetic approach. Chem. Asian. J. 2, 820-870 (2007).

40. Collins, K. D. \& Glorius, F. A robustness screen for the rapid assessment of chemical reactionsNat. Chem. 5, 597-601 (2013).

41. Grimme, S., Antony, J., Ehrlich, S. \& Krieg, H. A consistent and accurate ab initio parametrization of density functional dispersion correction (DFT-D) for the 94 elements $\mathrm{H}-\mathrm{Pu}$. J. Chem. Phys. 132, 154104 (2010).

42. Grimme, S., Ehrlich, S. \& Goerigk, L. Effect of the damping function in dispersion corrected density functional theory. J. Comput. Chem. 32, 1456-1465 (2011).

43. Frisch, M. J. et al. Gaussian 16, revision A.03, Gaussian, Inc. (2016).

44. Zhao, Y. \& Truhlar, D. G. Density functionals with broad applicability in chemistry. Acc. Chem. Res. 41, 157-167 (2008).

45. Zhao, Y. \& Truhlar, D. G. The M06 suite of density functionals for main group thermochemistry, thermochemical kinetics, noncovalent interactions, excited states, and transition elements: two new functionals and systematic testing of four M06-class functionals and 12 other functionals. Theor. Chem. Acc. 120, 215-241 (2008).

46. Cossi, M., Rega, N., Scalmani, G. \& Barone, V. Energies, structures, and electronic properties of molecules in solution with the C-PCM solvation model. J. Comput. Chem. 24, 669-681 (2003).

47. Barone, V. \& Cossi, M. Quantum calculation of molecular energies and energy gradients in solution by a conductor solvent model. J. Phys. Chem. A. 102, 1995-2001 (1998).

48. Grimme, S. et al. Fully automated quantum-chemistry-based computation of spin-spincoupled nuclear magnetic resonance spectra. Angew. Chem. Int. Ed. 56, 14763-14769 (2017).

49. Grimme, S. Exploration of chemical compound, conformer, and reaction space with metadynamics simulations based on tight-binding quantum chemical calculations. J. Chem. Theory Comput. 15, 2847-2862 (2019).

50. Grimme, S., Bannwarth, C. \& Shushkov, P. A robust and accurate tight-binding quantum chemical method for structures, vibrational frequencies, and noncovalent interactions of large molecular systems parametrized for all spd-block elements $(\mathrm{Z}=1-86)$. J. Chem. Theory Comput. 13,1989-2009 (2017). 
51. Bannwarth, C., Ehlert, S. \& Grimme, S. GFN2-xTB-An accurate and broadly parametrized self-consistent tight-binding quantum chemical method with multipole electrostatics and density-dependent dispersion contributions. J. Chem. Theory Comput. 15, 1652-1671 (2019).

52. Pracht, P., Caldeweyher, E., Ehlert, S. \& Grimme, S. A robust non-self-consistent tight-binding quantum chemistry method for large molecules. ChemRxiv, preprint. DOI:10.26434/chemrxiv.8326202.v1. (2019).

\section{Acknowledgements}

Financial support was provided by the Research Grants Council of Hong Kong (16302318, 16303420, 11303320, 11104020), the National Natural Science Foundation of China (91956114, 22077108), the Science and Technology Innovation Committee of Shenzhen (JCYJ20180507181627057), and Guangdong Provincial Key Laboratory of Catalysis (No. 2020B121201002), and the National Science Foundation (CHE-1764328 to K.N.H). Computational work was supported by Center for Computational Science and Engineering at Southern University of Science and Technology, and the Extreme Science and Engineering Discovery Environment (XSEDE), which is supported by the National Science Foundation (OCI-1053575). We also thank Dr. Herman H. Y. Sung and Dr. Ian D. Williams for help with structure elucidation.

\section{Author contributions}

Q.Y. conceived the project, performed the synthesis experiments, and wrote the paper. M.D. and P.Y. performed DFT calculations. C.C., Z.D. and M.W. performed the biological experiments. M.H. and G.Z. directed the biological experiments and data analysis. K.N.H. directed the DFT calculations and mechanism analysis. J.S. conceived and directed the project and wrote the paper. All the authors discussed the results and commented on the manuscript.

\section{Competing interests}

The authors declare no competing interests. 